\title{
The Study on the Flow Generated by an Array of Four Flettner Rotors: Theory and Experiment
}

\author{
Fernando Garzón1, Aldo Figueroa² \\ ${ }^{1}$ Instituto de Investigación en Ciencias Básicas y Aplicadas, Universidad Autónoma del Estado de Morelos, Cuernavaca, México \\ ${ }^{2}$ CONACYT-Centro de Investigación en Ciencias, Universidad Autónoma del Estado de Morelos, Cuernavaca, México \\ Email: alfil@uaem.mx
}

How to cite this paper: Garzón, F. and Figueroa, A. (2017) The Study on the Flow Generated by an Array of Four Flettner Rotors: Theory and Experiment. Applied Mathematics, 8, 1851-1858. https://doi.org/10.4236/am.2017.812132

Received: December 6, 2017

Accepted: December 26, 2017

Published: December 29, 2017

Copyright (C) 2017 by authors and Scientific Research Publishing Inc. This work is licensed under the Creative Commons Attribution International License (CC BY 4.0).

http://creativecommons.org/licenses/by/4.0/ cc) (i) Open Access

\begin{abstract}
We present an immersed array of four rotors whose promoted flow can be mathematically modeled with a creeping flow solution from the incompressible Navier-Stokes equations. We show that this solution is indeed representative of the two-dimensional experiment and validate such class of solution with experimental data obtained through the Particle Image Velocimetry technique and time-lapsed particles visualizations.
\end{abstract}

\section{Keywords}

Fluid Mechanics, Flettner Rotor, Fundamental Solutions

\section{Introduction}

Boats ships have crossed the oceans around the globe for centuries by the use of paddling, sails, engine propellers or even turbine propellers. An alternative to the latter propulsion systems is the rotor ship. Through this alternative technique, the ship is propelled, at least in part, by large vertical rotors, sometimes known as rotor sails. German engineer Anton Flettner was the first to build a ship which attempted to tap this force for propulsion, and the ships are sometimes known as Flettner ships [1]. A rotor ship is a type of ship designed to use the Magnus effect for propulsion, where the rotation of a solid body can modify its trajectory due to the frictional forces within fluids as consequence of its viscosity [2]. Flettner ships have arrays of large rotorsails that rise from its deck which are rotated via a mechanical linkage to the ship's propellers. Trials confirm fuel savings of 2.6 percent using a single small Rotor Sail on a route in the North Sea. With these fuel savings, this new wind propulsion technology has a payback period of just four years [3]. Flettner rotor propulsion system presents 
success in reducing fuel consumption and carbon dioxide $\left(\mathrm{CO}_{2}\right)$ emissions. Recent examples such as Enercon's E-ship 1 have proven seaworthy and economically viable along major shipping routes [4].

Recently, some preliminary assessments of numerical simulations have been conducted by comparison with experimental investigation of Flettner rotors in order to evaluate the functional relationship and the interaction between the control factors [5], the preliminary design of the Flettner rotor as a ships auxiliary propulsion system [6], its evaluation with another wind power technology, namely, the towing kite [7], and characterized in terms of lift and drag coefficient [8].

In contrast to the previous studies, in this article, a simple model derived from the Navier-Stokes equations is obtained and compared with a simple experimental model that represents the Flettner rotors from the Enercon's E-Ship 1, with four large rotor sails [9] when the ship is at rest and no flow is incident to it. The objective is the study of the behaviour of the flow patterns due to the rotating cylinders.

\section{Statement of the Problem}

The theoretical approach begins with the mass and momentum conservation for real fluids $[10]$

$$
\begin{gathered}
\nabla \cdot \boldsymbol{u}=0 \\
\frac{\partial \boldsymbol{u}}{\partial t}+(\boldsymbol{u} \cdot \nabla) \boldsymbol{u}=-\frac{1}{\rho} \nabla p+v \nabla^{2} \boldsymbol{u}
\end{gathered}
$$

where the velocity vector is denoted by $\boldsymbol{u}, \quad p$ is the pressure, $\rho$ is the density, $v$ is the kinematic viscosity and $t$ represents time. Considering that the surface is flat, and that the motions is laminar, thus the motion of the flow occurs on the $x-y$ plane and the perpendicular velocity is negligible, thus we assume that the flow is two-dimensional. As a first approach, we consider a single rotor. Locating the origin in the geometrical center of the rotor and using polar coordinates, the flow can be considered as symmetric around the origin, that is, independent of the $\theta$-direction. Thus the velocity vector is $\boldsymbol{u}=u_{\theta}(r)$, which automatically satisfies the continuity Equation (1) and the Navier-Stokes Equation (2) for the $r$ and $\theta$ components are

$$
\begin{gathered}
\frac{u_{\theta}^{2}}{r}=\frac{1}{\rho} \frac{\mathrm{d} p}{\mathrm{~d} r} \\
\frac{\mathrm{d}^{2} u_{\theta}}{\mathrm{d} r^{2}}+\frac{\mathrm{d}}{\mathrm{d} r}\left(\frac{u_{\theta}}{r}\right)=0
\end{gathered}
$$

Equation (4) is a homogeneous second order differential equation, thus we must impose two boundary conditions. The first one is that the velocity at infinity is zero, that is $u_{\theta}(\infty)=0$. The second one is a non-slip condition, as the cylinder rotates with a uniform angular velocity $\omega$, the tangential velocity at the cylinder's radius $R_{i}$ is: $u_{\theta}\left(R_{i}\right)=\omega R_{i}$, where $\omega=2 \pi f$ and $f$ is the rotating cylinder frequency. The solution to Equation (4) is 


$$
u_{\theta}(r)=\frac{\omega R_{i}^{2}}{r}
$$

The stream lines can be obtained with the following relation

$$
\frac{\mathrm{d} \psi}{\mathrm{d} r}=u_{\theta}
$$

where $\psi(r)$ is the stream function. The boundary condition is $\psi\left(R_{i}\right)=0$, so we get

$$
\psi(r)=\omega R_{i}^{2}\left(\ln (r)-\ln \left(R_{i}\right)\right)
$$

Once the velocity field is known, the pressure distribution can be calculated from Equation (3)

$$
p(r)=-\frac{\rho \omega^{2} R_{i}^{4}}{2 r^{2}}+C
$$

where $C$ is a constant that can be obtained by evaluating $p(\infty)$. The solution (5) and stream line function (7) in Cartesian coordinates are

$$
\begin{gathered}
u(x, y)=\frac{\omega R_{i}^{2}}{\sqrt{x^{2}+y^{2}}} \cos (\arctan (y / x)) \\
v(x, y)=\frac{\omega R_{i}^{2}}{\sqrt{x^{2}+y^{2}}} \sin (\arctan (y / x)) \\
\psi(x, y)=\omega R_{i}^{2}\left(\ln \left(\sqrt{x^{2}+y^{2}}\right)-\ln \left(R_{i}\right)\right)
\end{gathered}
$$

As stated previously, Equations (5) and (7) correspond to single rotor located at the origin. However, as the solution is linear, we can sum the solutions to consider the flow of four rotating cylinders, that is we can perform a superposition of rotors. In Cartesian coordinates, the total stream lines solutions $\Psi$ for the four contemplated sets of rotation are as follows:

$$
\begin{aligned}
& \Psi_{1}(x, y)=\psi(x+a, y)+\psi(x, y+a)+\psi(x-a, y)+\psi(x, y-a) \\
& \Psi_{2}(x, y)=\psi(x+a, y)+\psi(x, y+a)-\psi(x-a, y)+\psi(x, y-a) \\
& \Psi_{3}(x, y)=-\psi(x+a, y)+\psi(x, y+a)-\psi(x-a, y)+\psi(x, y-a) \\
& \Psi_{4}(x, y)=-\psi(x+a, y)-\psi(x, y+a)+\psi(x-a, y)+\psi(x, y-a)
\end{aligned}
$$

where the function $\psi$ represents the flow generated by a single rotor with negative (clockwise) or positive (counter clockwise) rotation depending on its sign, and $a$ is the shifting length from the origin. Next sections show the experimental procedure and the comparison between this theoretical model and the experimental measurements.

\section{Experimental Procedure}

The experiment set up consist of four rotating cylinders immersed in a fluid layer. The working fluid is water at room temperature $\left(20^{\circ} \mathrm{C}\right)$. The fluid is contained in a $30 \times 30 \times 10 \mathrm{~cm}$ glass box which is open in the upper side, see Figure 1. The measures of the box were calculated so that its boundaries are 


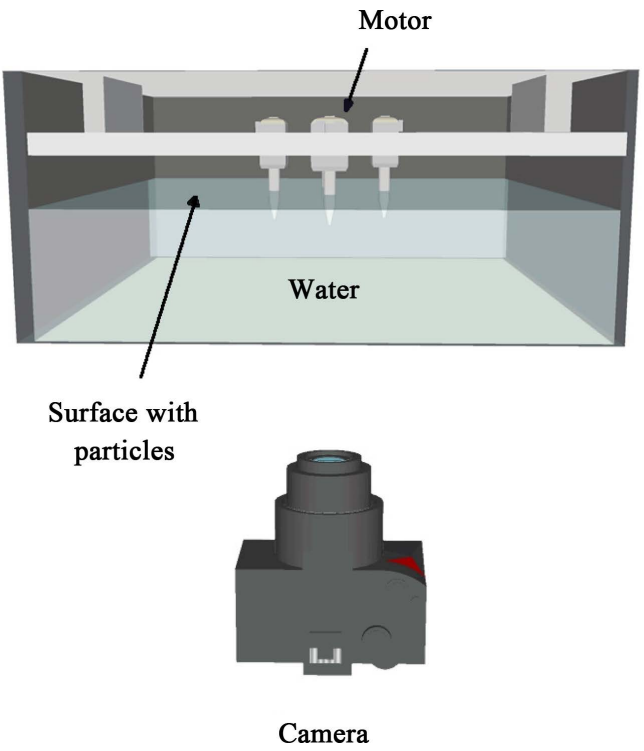

(a)

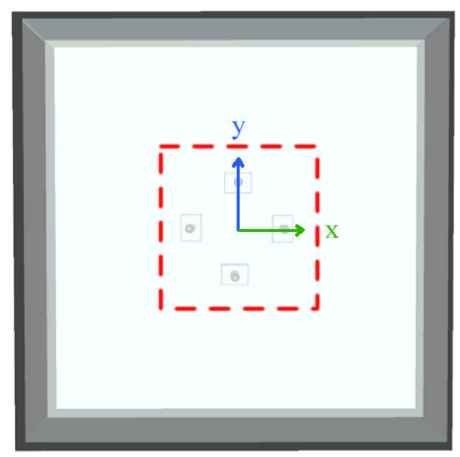

Captured

zone

(b)

Figure 1. Sketch of the experimental set-up, not drawn to scale. (a) Lateral view; (b) Visualized $x-y$ plane. The flow promoted by the rotors is visualized in the $x$ - $y$ plane thorough the PIV technique.

sufficiently far away from the flow. The height of the water level in the glass container is $8 \mathrm{~cm}$. Above the fluid, a rigid table is placed at the top of the box with the four electric DC motors (6 V with gearbox) embedded in it. Considering the geometric center of the rigid table as the origin of coordinates, the electric motors are located at a distance of $2.5 \mathrm{~cm}$ from the origin. A rigid cylinder $L=10 \mathrm{~cm}$ is coupled to the shaft of every motor so that the cylinder is partially submerged in the liquid. The radius of every cylinder is $R_{i}=0.4 \mathrm{~cm}$. The DC motors are connected to a power supply whose output voltage is $6 \mathrm{~V}$. As a single cylinder rotates, it promotes a circular fluid flow around itself. The direction of rotation of the cylinders can be changed by inverting the polarity of each motor. As every cylinder rotates independently, four sets of rotation are studied. Every set of rotation produces a different flow pattern. The frequency of rotation of the rotors is $f=0.667 \mathrm{~Hz}$. The surface of the fluid is seeded with hollow glass spheres, with an approximated diameter of $10 \mu \mathrm{m}$. Images of the surface are recorded with a photographic camera Nikon D90 with a AF micro-nikkor $60 \mathrm{~mm}$ $\mathrm{f} / 2.8 \mathrm{D}$ lens placed underneath the glass box. Streamlines of the flow cases are obtained by using an exposition time of $30 \mathrm{~s}$. The Particle Image Velocimetry (PIV) technique was used in order to obtain velocity vector fields. The experimental data gathered was used to perform a comparative with the theoretical results.

\section{Main Results}

In this section, the experimental results are compared with the simple theoretical model obtained from the Navier-Stokes equations. Figure 2 and Figure 3 show 


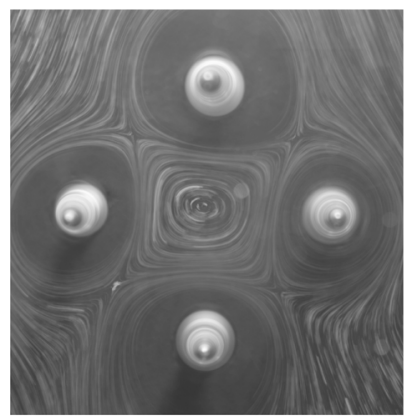

(a)

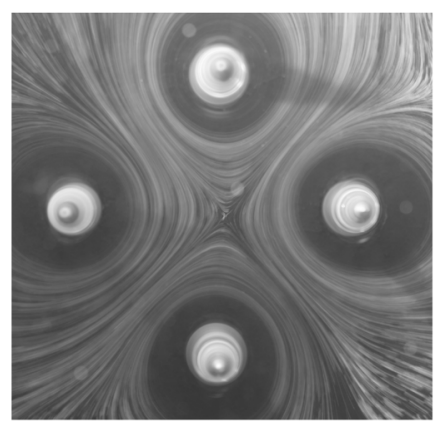

(c)

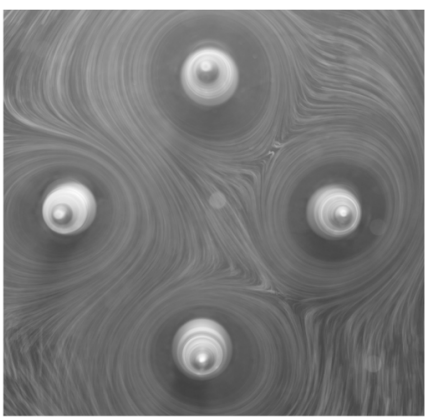

(b)

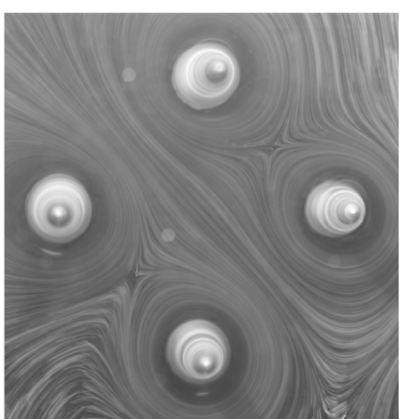

(d)

Figure 2. Stream lines of the flows generated by the four sets of rotation. As defined in Equation (12): (a) Set of rotation 1; (b) Set of rotation 2; (c) Set of rotation 3; (d) Set of rotation 4. Experimental results.

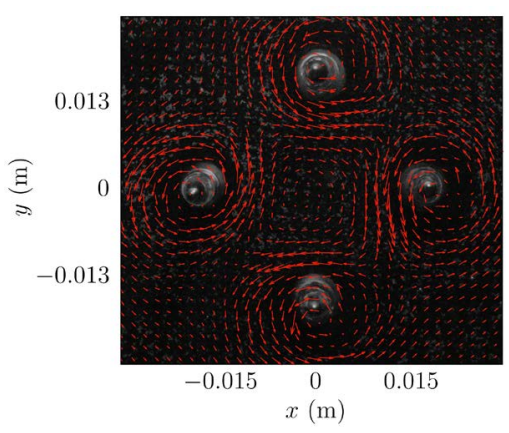

(a)

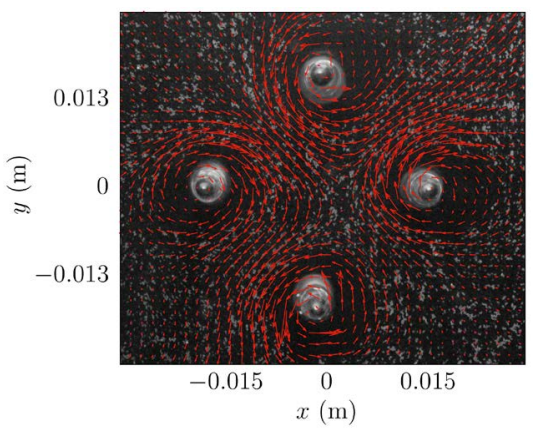

(c)

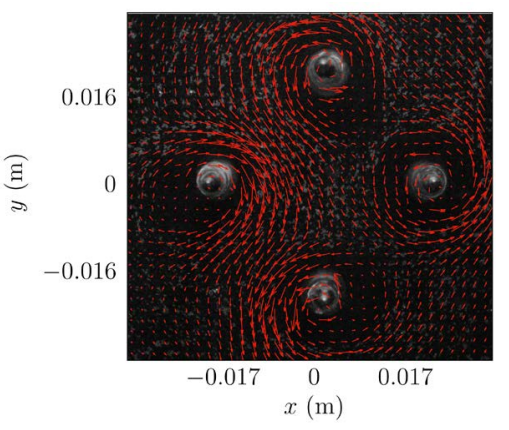

(b)

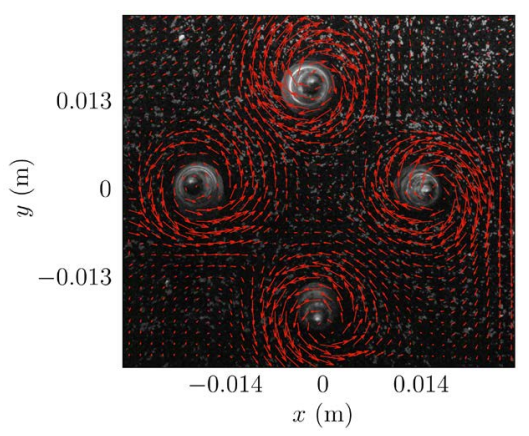

(d)

Figure 3. Velocity field of the flows generated by the four sets of rotation. As defined in Equation (12): (a) Set of rotation 1; (b) Set of rotation 2; (c) Set of rotation 3; (d) Set of rotation 4. Experimental results. 
the experimental stream lines and the vector fields, respectively, for the four sets of rotation. The stream lines allow to easily identify the flow patterns. The first case, Figure 2(a), is characterized by a squared inner vortex among the four rotors sharing a horseshoe at every corner. The second case, Figure 2(b), is an asymmetric flow with two horseshoe patterns. Whereas the third case, Figure 2 (c), is a large horseshoe pattern in the geometrical centre. Finally, the fourth case, Figure 2(d), is a jet flow from low-right corner to top-left corner. In turn, the vectorial fields allow us to identify the direction of the flows and their magnitude. Moreover, we can appreciate that every cylinder generates a vortex patter around itself and the interaction of the four vortices rotation in different directions produce a global flow. As the theoretical model departs from the latter assumption, its predictions are close to the experimental results as seen in Figure 4, where the stream lines from Equation (12) are shown with $a=25 \mathrm{~mm}$. Comparing with Figure 2, only the first case does not agree completely, since the inner squared vortex is dismissed by the analytical solution. However, for the rest of the cases the location of the horseshoes, the vortices and the jets are completely predicted. Even more, Figure 5 compares the experimental velocity profiles with the theoretical predictions from Equation (9) for flow cases 1 and 4, see Equation (12). This is a more detailed comparison and even at this level the qualitative comparison is very good. The Reynolds number for the experiments

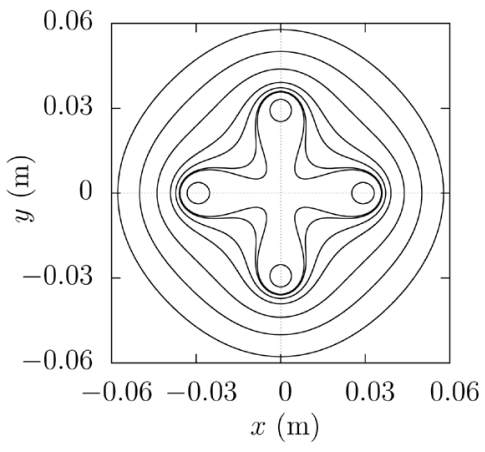

(a)

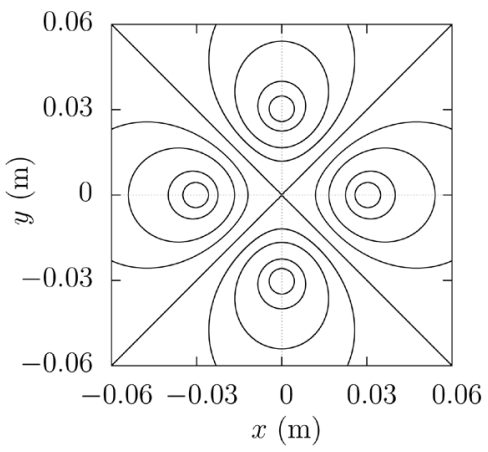

(c)

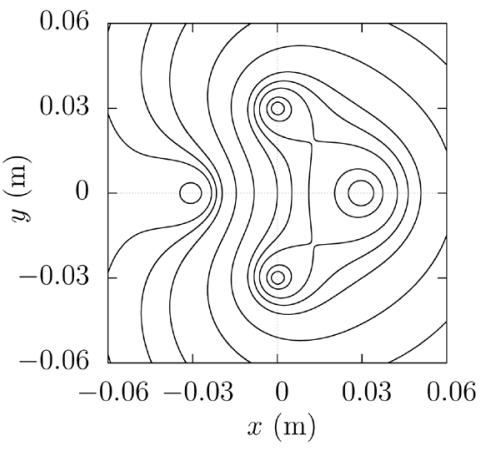

(b)

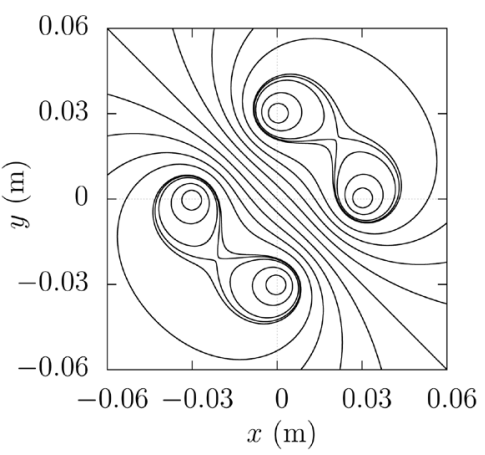

(d)

Figure 4. Stream lines of the flows generated by the four sets of rotation. As defined in Equation (12): (a) Set of rotation 1; (b) Set of rotation 2; (c) Set of rotation 3; (d) Set of rotation 4 . Theoretical calculations. 


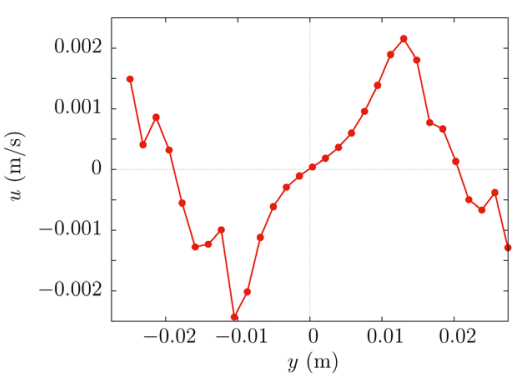

(a)

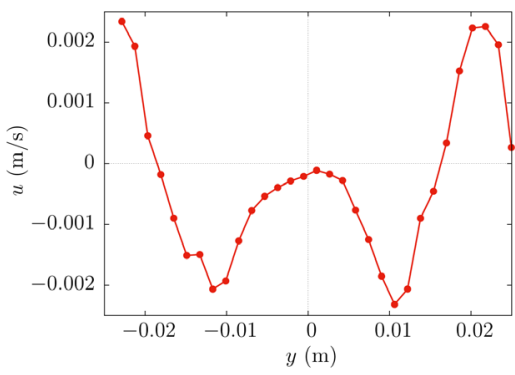

(c)

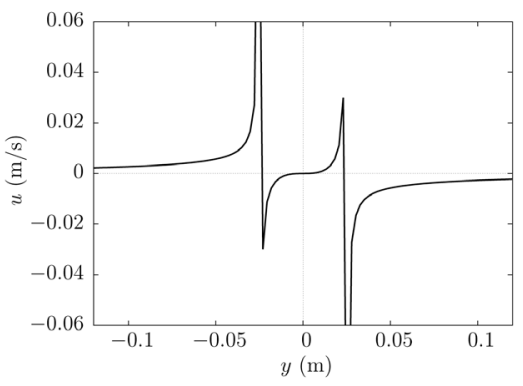

(b)

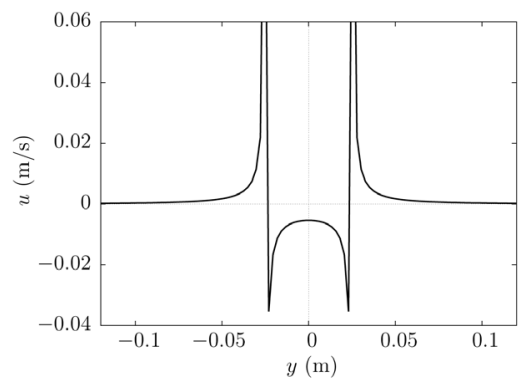

(d)

Figure 5. Horizontal velocity $u$ as a function of the $y$-direction. (a) and (c) experimental profiles of the sets of rotation 1 and 4, respectively; (b) and (d) theoretical profiles of the sets of rotation 1 and 4 , respectively.

is $R e=U R_{i} / v=8$, where $U$ is the maximum velocity in the flow field, which demonstrates that the flow regime is laminar and can be compared with the theoretical model. The theoretical predictions are one order of magnitude higher than experimental measurements. This can be attributed to the friction of the container's bottom with the fluid. Since the model is two-dimensional this friction is not taken into account, thus the velocity in the theoretical model is higher than the experimental obtained through PIV.

\section{Conclusions and Suggestions}

A solution for the incompressible Navier-Stokes equations was derived in the creeping regime for the case of a single rotation rotor. Considering its linearity, it was superposed to consider an array of four rotors in an unbounded domain. An investigation of the validity of this solution was carried out experimentally. It turned out that this solution represents qualitatively the experimental problem. A verification was established through PIV and time-lapse particles visualization.

\section{Acknowledgements}

This research was supported by CONACYT, Mexico, under project 258623. A.F. thanks the Cátedras program from CONACYT.

\section{References}

[1] Neu Wayne, L. (2013) Flettner Rotor Ship. AccessScience McGraw-Hill Education. 
https://doi.org/10.1036/1097-8542.YB130204

[2] Miller, F.P., Vandome, A.F. and John, M.B. (2010) Magnus Effect. https://books.google.com.mx/books?id=SAPIXwAACAAJ

[3] Marks, H. and Wachtel, B. (2015) Ship Efficiency Technologies Ready to Set Sail. https://www.greenbiz.com/article/ship-efficiency-technologies-ready-set-sail

[4] Searcy, T. (2017) Harnessing the Wind: A Case Study of Applying Flettner Rotor Technology to Achieve Fuel and Cost Savings for Fiji's Domestic Shipping Industry. Marine Policy, 86, 164-172. https://doi.org/10.1016/j.marpol.2017.09.020

[5] De Marco, A., Mancini, S., Pensa, C., Scognamiglio, R. and Vitiello, L. (2015) Marine Application of Flettner Rotors: Numerical Study on a Systematic Variation of Geometric Factor. VI International Conference on Computational Methods in Marine Engineering, Rome, 1-12.

[6] De Marco, A., Mancini, S., Pensa, C., Calise, G. and De Luca, F. (2016) Flettner Rotor Concept for Marine Applications: A Systematic Study. International Journal of Rotating Machinery, 2016, 1-12. https://doi.org/10.1155/2016/3458750

[7] Traut, M., Gilbert, P., Walsh, C., Bows, A., Filippone, A., Stansby, P. and Wood, R. (2013) Propulsive Power Contribution of a Kite and a Flettner Rotor on Selected Shipping Routes. Applied Energy, 13, 362-372.

[8] Gadkari, M., Deshpande, V., Mahulkar, S., Khushalani, V., Pardhi, S. and Kedar, A.P. (2017) To Study Magnus Effect on Flettner Rotor. International Research Journal of Engineering and Technology, 4, 1597-1601.

[9] Wikipedia (2017) E-Ship 1. https://en.wikipedia.org/wiki/E-Ship1

[10] Currie, I.G. (1993) Fundamental Mechanics of Fluids. Mechanical Engineering, Marcel Dekker, New York. 